\title{
Nanomedicine and drug delivery: a mini review
}

\author{
Agha Zeeshan Mirza $\cdot$ Farhan Ahmed Siddiqui
}

Received: 24 October 2013 / Accepted: 23 January 2014/Published online: 20 February 2014

(c) The Author(s) 2014. This article is published with open access at Springerlink.com

\begin{abstract}
The field of nanotechnology now has pivotal roles in electronics, biology and medicine. Its application can be appraised, as it involves the materials to be designed at atomic and molecular level. Due to the advantage of their size, nanospheres have been shown to be robust drug delivery systems and may be useful for encapsulating drugs and enabling more precise targeting with a controlled release. In this review specifically, we highlight the recent advances of this technology for medicine and drug delivery systems.
\end{abstract}

Keywords Drug delivery - Nanotechnology · Inorganic nanoparticles $\cdot$ Organic nanoparticles

\section{Nanoparticles}

Nanoparticles are organic or inorganic structures (sizes 1-100 nm) similar to antibodies and DNA plasmids [1-3]. Significant work has been done in past decades in the field of nanotechnology; now it is possible to fabricate, characterize, and modify the functional properties of nanoparticles for medical diagnostics and biomedical applications [4-10]. Nanobiotechnology bridges the physical and biological sciences [11], with applications of nanophase and nanostructures in different areas of science, especially in biomedicine, in which these objects are of great interest

\footnotetext{
A. Z. Mirza ( $\square)$

Department of Chemistry, University of Karachi, Karachi 75270,

Pakistan

e-mail: dr.zeeshan80@gmail.com

F. A. Siddiqui

Faculty of Pharmacy, Federal Urdu University of Arts, Science and Technology, Karachi 75300, Pakistan
}

[12-16]. Nanoparticles are influencing the field of medicine from nanobiotechnology, microfluidics, biosensors, drug delivery, and microarrays to tissue micro-engineering [17]. Metal, organic and polymeric nanoparticles and liposomes are commonly studied and can be designed for the site-specific delivery of drugs (Fig. 1) [18] especially those drugs which have poor solubility and absorption $[19,20]$.

\section{Nanoparticles for drug delivery}

The most promising application of nanomaterials is the promise of targeted, site-specific drug delivery. The potential of eliminating a tumorous outgrowth without any collateral damage through nanomaterial-based drug delivery has created significant interest and nanoparticles form the basis for bio-nano-materials [3] and major efforts in designing drug delivery systems are based on functionalized nanoparticles [21, 22]. Initially, they were devised as carriers for vaccines and anticancer drugs [23] and then the nanometer size ranges may significantly enhance the drug delivery by affecting the bio-distribution and toxicodynamics of drugs [24, 25]. This can make in vivo delivery of many types of drugs which pose serious delivery problems, a relatively easy task [26]. Modifying or functionalizing nanoparticles to deliver drugs through the blood brain barrier for targeting brain tumors can be regarded as a brilliant outcome of this technology [27]. For example, doxorubicin does not cross the blood-brain barrier, but its integration with polysorbate 80 modified polybutylcyanoacrylate nanoparticles can increase its delivery to the brain to a significant extent [28]. Due to their size, shape and functionality, nanoparticle systems play a pivotal role in creation of DNA delivery vectors [29]. They can penetrate deep into tissues and are absorbed by the cells efficiently 


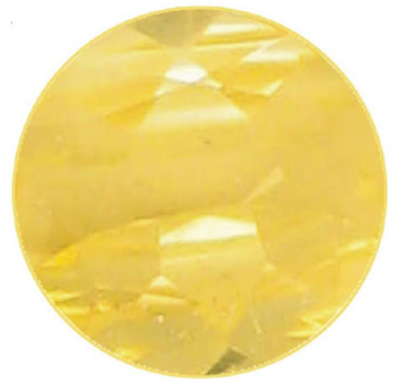

Metal nanoparticles

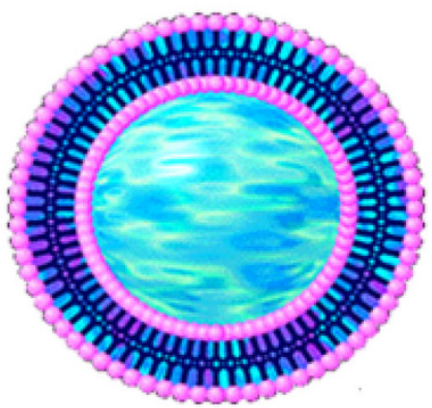

Liposomes

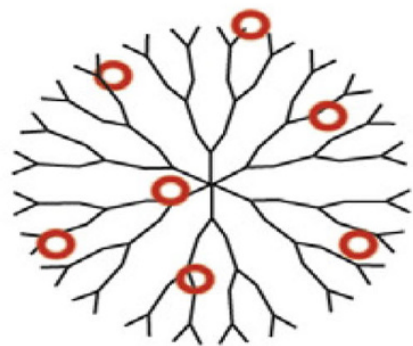

Dendrimers

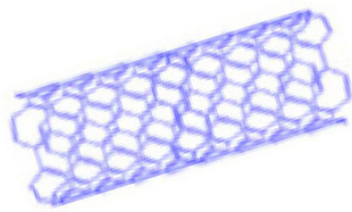

Carbon nano tubes

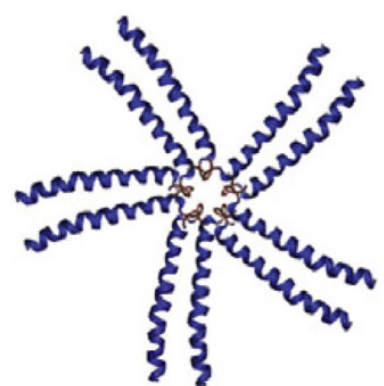

Peptide based nanoparticles

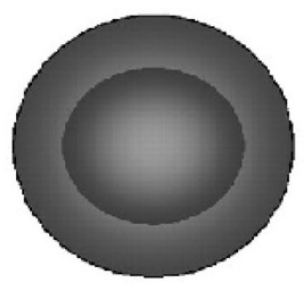

Quantum Dots

Fig. 1 Example of metal nanoparticles, dendrimers, peptide-based nanoparticles, liposomes, carbon nanotubes and quantum dots

[30]. Nano-sized colloidal carriers of drugs can be regarded as an advanced development in pharmacotherapy [31]. They act as potential carriers for several classes of drugs like anti-cancer, anti-hypertensive and hormones, etc. [18]. Submicron colloidal particles have been used as nanoparticles for the purpose of drug delivery [32] and also used for the diagnosis of diseases [26]. Nanoparticles have widened the scope of pharmacokinetics for insoluble drugs. For example, the trans-retinoic acid nanoparticle coated by $\mathrm{CaCO}_{3}$ was developed as a new drug delivery system, which on spray drying formed aggregates. The aggregates thus formed were found to re-disperse in water, which stimulated insulin secretion from islets [33]. Generally, nanoparticle may be composed of polymeric or inorganic materials. Some important examples from literature are reviewed in the following sections.

\section{Polymeric nanoparticle}

Polymeric nanoparticles are made from biocompatible and biodegradable materials and a variety of natural (gelatin, albumin) and synthetic polymers (polylactides, polyalkylcyanoacrylates) [32] are considered as the most promising drug carrier as compared to liposomes [34].
Polymers as host material play a significant role in metals [34-36] and semiconductors [37-40] nanoparticles. These nanoparticles are designed as drug carrier with the objective of delivering active molecules to the intended target [41]. Polymers are filled with dispersed nano-fillers (smaller than $100 \mathrm{~nm}$ ) in nano-composites [42]. Polymer hydrophobicity, nanoparticle area and monomer concentration affect the adsorption capacity of the drug [43]. During polymerization, drugs may be added and be entrapped within the nanoparticle polymer network. Problems of drug bioavailability can be solved with nanotechnology. To improve drug absorption and bioavailability of hydrophobic drugs (paclitaxel or 5-fluorouracil) nano-scale cavities with liposomes or encapsulated polymers can be designed which can metabolize drugs at optimum rates for desired therapeutic effect in target tissues [44-46]. Nanocapsules can be synthesized using polymers or from albumin and liposomes [24].

There are several polymers available for the synthesis of nanoparticles. Polylactide-polyglycolide copolymers, polyacrylates and polycaprolactones, etc. are synthetic polymers whereas albumin, gelatin, alginate, collagen and chitosan used as natural polymers [25]. Polylactides and poly (DL-lactide-co-glycolide) polymers undergo hydrolysis upon implantation, form biologically compatible 
fragments, and are mostly investigated for drug delivery [47]. It is apparent that chemical conjugation of drugs with different polymers provides opportunities to increase their activities. Jie et al. synthesized amphiphilic $N$-(2-hydroxy)propyl-3-trimethylammonium-chitosan-cholic acid polymers by joining cholic acid and glycidyl trimethyl ammonium chloride onto chitosan and self-assembled into nanoparticles in phosphate-buffered saline. Doxorubicin could be encapsulated into these nanoparticles and then could easily be uptaken by breast cancer (MCF-7) cells and released into the cytoplasm [48]. Shengtang et al. synthesize folic acid-conjugated chitosan-polylactide copolymers to build a drug carrier with active targeting of paclitaxel. Targeting characteristic was confirmed using folic acid receptor-expressed MCF-7 breast cancer cells [49]. One research described the fabrication of quatemized poly(propylene imine) dendrimer of generation-3, QPPI (G3) as a drug carrier for poorly soluble anti-inflammatory drug nimesulide and an improvement in solubility of drug was observed [50]. Abdullah et al. [51] encapsulated nanoscaled emulsion containing ibuprofen with carbopol 934, 940 and ultrez 10 as viscosity modifiers. Behbehani et al. [52] observed the effect of silica nanoparticles on the activity of $\alpha$-amylase. Park et al. [53] reported that glycol chitosan-based nanoparticles of adriamycin are useful for sustained and specific delivery of adriamycin, thus showing lower cytotoxicity than adriamycin alone.

Release of drugs from nanoparticles and biodegradation are important parameters to be considered for developing successful formulations. Therefore, the diffusion characteristics and breakdown properties of the nanoparticles at the drug delivery site need to be carefully mapped to achieve effective therapeutic capabilities [54].

\section{Inorganic nanoparticles}

Nanoparticles offered important multifunctional platforms for biomedical applications. Varieties of nanoparticles, such as silica nanoparticles [55], quantum dots [56, 57], metal nanoparticles [58] and lanthanide nanoparticles [59, 60], have unique properties which are adapted for different applications in the bio-analysis field.

A nanoparticle does not only indicate drug delivery but confirmation of target delivery is also important. Tracking of nanomedicine from the systemic to sub-cellular level becomes essential. Many florescent markers are available; however, nanoparticles have not only advantage of showing improvement in fluorescent markers for medical imaging and diagnostic applications but also in imaging of tumors and other diseases in vivo [61]. For example, Lee et al. synthesized $\mathrm{Fe}_{3} \mathrm{O}_{4}$ nanocrystals on uniform dyedoped mesoporous silica nanoparticles to be used as a contrast agent in magnetic resonance imaging and loaded doxorubicin in the pores. This system has great potential as probes in magnetic resonance and fluorescence imaging and doxorubicin was successfully delivered to the tumor sites and its anticancer activity was retained [62]. Similarly, histidine-tagged cyan fluorescent protein-capped magnetic mesoporous silica nanoparticles system was fabricated for drug delivery and fluorescent imaging [63]. Quantum dots are small-sized $(1-10 \mathrm{~nm})$ semiconductor nanocrystals composed of inorganic elemental core (e.g., $\mathrm{Cd}$ and $\mathrm{Se}$ ) surrounded by a metallic shell $(\mathrm{ZnS})$. They are widely used in biological research and can also be used as drug carriers or simply as fluorescent labels for other drug carriers [56, 57]. Among the broad diversity of nanoparticles, iron oxide and gold nanoparticles are the most intensively studied [64]. Due to the presence of surface plasmons, gold, copper and silver nanoparticles strongly absorb light in the visible region, making it possible to study their size-dependent light absorption through surface plasmon resonance (SPR). Gold nanoparticles and nanorods have many unique properties, which have been explored for potential applications in bio-molecular detection (Fig. 2) [65]. In terms of biocompatibility and non-cytotoxicity, gold nanoparticles as approved by the FDA have distinct advantages over other metallic particles and could also be utilized as a favorable carrier for delivery of drugs [66]. These nanoparticles can be conjugated with amino acid [67] and proteins [68]. Fabrication of gold nanoparticles and functionalization with organic molecules to interact with any physiological system are more important [69-71]. These functionalized nanoparticles are a promising candidate for drug delivery as biomarkers of drug resistance cancer cell [72]. Reported application of gold nanoparticles includes insulin delivery by nasal route [73], improved antimicrobial action against E. coli strains [74] and ciprofloxacin-protected nanoparticles [75] for better drug release. Dhar et al. [64] reported a method for synthesis of gold nanoparticles using natural, gellan gum for the delivery of doxorubicin hydrochloride and demonstrated the successful loading of doxorubicin onto gold nanoparticles. Similarly, Gibson et al. [76] defined a very accurate measurement of biological activity by welldefined preparation of gold drug nanoparticle system. Hwu et al. [72] synthesized three paclitaxel-conjugated nanoparticles using $\mathrm{Fe}_{3} \mathrm{O}_{4}$ and gold as the core. These conjugated nano-materials comprise a new class of candidates as anticancer drugs. Our group has investigated synthesis and functionalization of gold nanoparticles [77]. We have functionalized gold nanoparticles with different anticancer drugs for target drug delivery and also as reducing and capping agents for gold nanoparticle synthesis. As such, the applications are very broad and useful for the release of different biologically active molecules. Recent work by us 
Fig. 2 a TEM images of gold nanoparticles, b nanorods,

c SEM images of nanoparticles

Fig. 3 Visible-NIR spectra of gold nanoparticles (a) and nanorods (b)
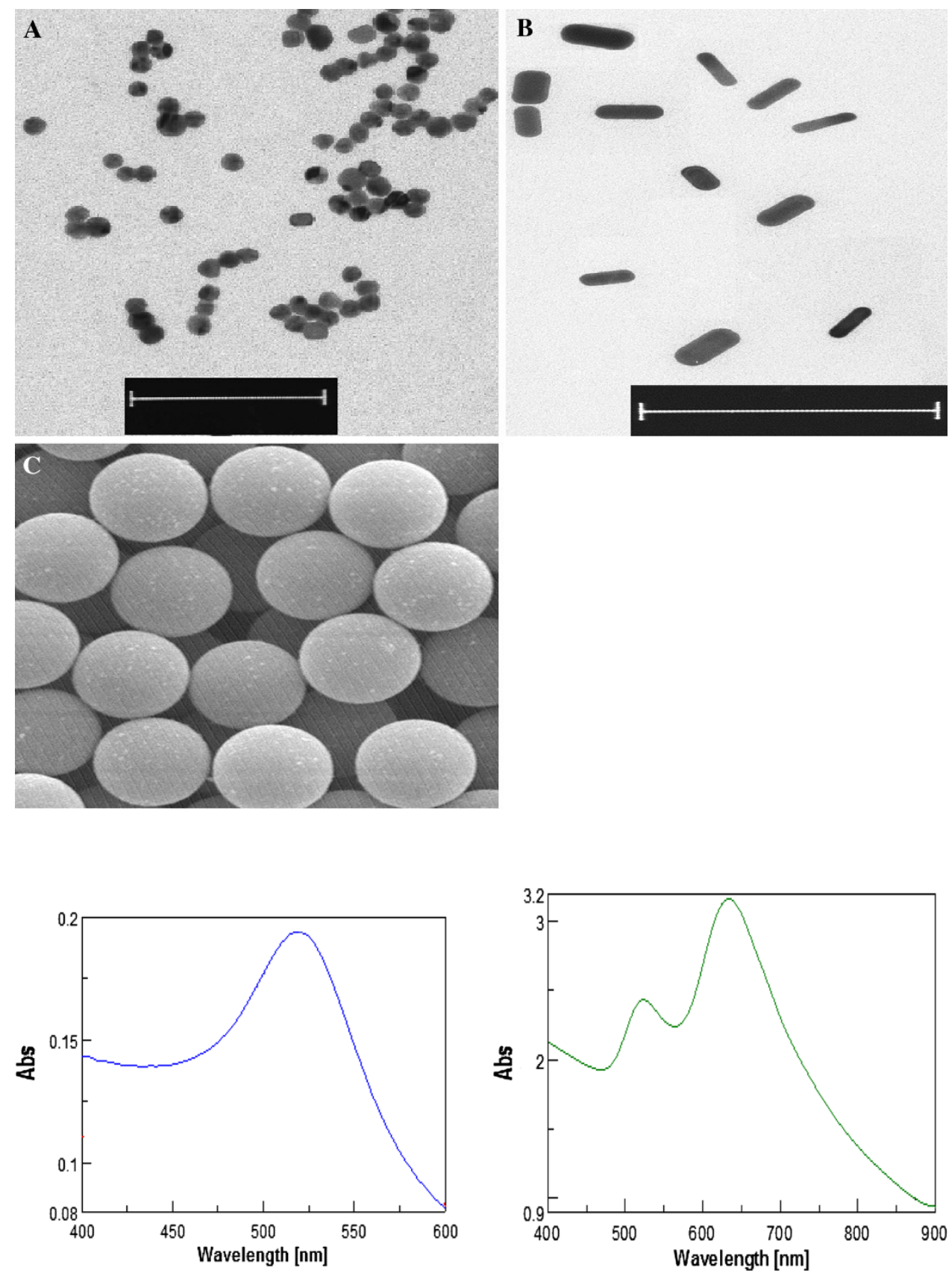

suggested that Au NPs-Au NRs complexes can also be used for further attachment of drugs and biomolecules for potential-targeted drug delivery [78]. Our research indicated that the surface modification can enhance the stability of Au NRs and we can use Au NRs as multiplex biosensors. Our focus was on the development of nanoparticles for bio-sensing and targeted drug delivery for cancer therapeutics. We developed folic acid and doxorubicintagged nanoparticles through a novel chemical linking protocol and tagged folic acid and doxorubicin on different planes of the nanorods. In this configuration, the nanorods can potentially target cancer cells very selectively and then deliver a cancer drug with high efficiency. An investigation of biological activity of this system is ongoing and will be reported.

Nanoparticle characterization can be performed by measuring the following parameters as particle size, surface charge, surface functionality and optical and magnetic properties. Formation of nanoparticles is demonstrated using surface plasmon resonance by the UV-Vis-NIR absorption spectra (Fig. 3). The most commonly used techniques to measure nanoparticles size are transmission electron microscopy (TEM) and scanning electron microscopy (SEM). The zeta potential of a particle is used 
to establish the colloidal stability of nanoparticles and it also gives the complete picture of overall charges. Moreover, it can also help in formulating the more stable nanoparticles product by shedding information on the effect of various parameters as $\mathrm{pH}$, concentration of an additive or ionic strength of the medium on it $[79,80]$.

\section{Conclusion}

Nanoparticles are rapidly becoming the focus of most efforts aiming at targets and site-specific drug delivery. The targeting ability of nanoparticles depends on certain factors such as particle size, surface charge, surface modification and hydrophobicity. Still many problems related to selective binding, targeted delivery and toxicity need to be overcome. Limited knowledge about the toxicity of nanoparticles is a major concern and certainly deserves more attention. If these nanoparticles are cautiously designed to tackle problems related to target and route of administration, they may lead to a new more successful paradigm in the world of therapeutics and research. The most promising research in nanoparticle production is via using supercritical fluids which are environmental friendly and free of toxic solvents. Much research is currently being performed to overcome these hurdles which will definitely establish nanoparticle-based drug delivery as the gold standard for site-specific therapeutics.

Conflict of interest The authors declare that they have no competing interests.

Authors' contributions AZM surveyed and drafted the review article. All authors read and approved the final review.

Open Access This article is distributed under the terms of the Creative Commons Attribution License which permits any use, distribution, and reproduction in any medium, provided the original author(s) and the source are credited.

\section{References}

1. Whitesides, G.M.: The 'right' size in nanobiotechnology. Nat. Biotech. 21, 1161-1165 (2003)

2. Lowe, C.R.: Nanobiotechnology: the fabrication and applications of chemical and biological nanostructures. Curr. Opin. Chem. Biol. 10, 428-434 (2000)

3. Wang, L., Zhao, W., Tan, W.: Bioconjugated silica nanoparticles: development and applications. Nano Res. 1, 99-115 (2008)

4. John, V.T., Simmons, B., McPherson, G.L., Bose, A.: Recent developments in materials synthesis in surfactant systems. Curr. Opin. Colloid Interface Sci. 7, 288 (2002)

5. Castelvetro, V., De Vita, C.: Nanostructured hybrid materials from aqueous polymer dispersions. Adv. Colloid Interface Sci. 108-109, 167-185 (2004)

6. Jang, J.H., Shea, L.D.: Controllable delivery of non-viral DNA from porous scaffolds. J Control Release 86, 157-168 (2003)
7. Shi, M., Yang, Y.Y., Chaw, C.S., Goh, S.H., Moochhala, S.M., Ng, S., Heller, J.: Double walled POE/PLGA microspheres: encapsulation of water-soluble and water-insoluble proteins and their release properties. J. Control Release 89, 167-177 (2003)

8. Mu, L., Feng, S.S.: Fabrication, characterization and in vitro release of paclitaxel (taxol) loaded poly (lactic-co-glycolic acid) microspheres prepared by spray drying technique with lipid/cholesterol emulsifiers. J. Control Release 76, 239-254 (2001)

9. Jones, C.D., Fidalgo, M.M., Wiesner, M.R., Barron, A.R.: Alumina ultrafiltration membranes derived from carboxylate-alumoxane nanoparticles. J. Membr. Sci. 193, 175-184 (2001)

10. Gupta, A.K., Gupta, M.: Synthesis and surface engineering of iron oxide nanoparticles for biomedical applications. Biomaterials 26, 3995-4201 (2005)

11. Liu, Z., Tabakman, S., Welsher, K., Dai, H.: Carbon nanotubes in biology and medicine: in vitro and in vivo detection, imaging and drug delivery. Nano Res. 2, 85-120 (2009)

12. Razzacki, S.Z., Thwar, P.K., Yang, M., Ugaz, V.M., Burns, M.A.: Integrated microsystems for controlled drug delivery. Adv. Drug Deliv. Rev. 56, 185-198 (2004)

13. Frank, A., Kumar, R.S., Boey, F., Venkatraman, S.: Study of the initial stages of drug release from a degradable matrix of poly(D, L-lactide-co-glycolide). Biomaterials 25, 813 (2004)

14. Freitas, R.A.: What is nanomedicine? Nanomedicine 1, 2-9 (2005)

15. Vinogradov, S.V., Bronich, T.K., Kabanov, A.V.: Nanosized cationic hydrogels for drug delivery: preparation, properties and interactions with cells. Adv. Drug Deliv. Rev. 54, 135-147 (2002)

16. Orive, G., Gascón, A.R., Hernández, R.M., Gil, A.D., Pedraz, J.L.: Techniques: new approaches to the delivery of biopharmaceuticals. Trends Pharmacol. Sci. 25, 382-387 (2004)

17. Arayne, M.S., Sultana, N., Qureshi, F.: Review: nanoparticles in delivery of cardiovascular drugs. Pak. J. Pharm. Sci. 20, 340-348 (2007)

18. Bala, I., Hariharan, S., Kumar, M.N.: PLGA nanoparticles in drug delivery: the state of the art. Crit. Rev. Ther. Drug Carrier Syst. 21, 387-422 (2004)

19. Karen, K., Tasana, P., Nigel, D.M., Thomas, R.: Entrapment of bioactive molecules in poly (alkylcyanoacrylate) nanoparticles. Am. J. Drug Deliv. 4, 251-259 (2004)

20. Reddy, L.H., Murthy, R.S.R.: Pharmacokinetics and biodistribution studies of doxorubicin loaded poly(butyl cyanoacrylate) nanoparticles synthesized by two different techniques. Biomed. Pap. Med. Fac. Univ. Palacky Olomouc Czech Repub. 148, 161-166 (2004)

21. Yokoyama, M., Miyauchi, M., Yamada, N., Okano, T., Sakurai, Y., Kataoka, K., Inoue, S.: Polymer micelles as novel drug carrier: adriamycin-conjugated poly(ethylene glycol)-poly(aspartic acid) block copolymer. J. Control. Rel. 11, 269 (1990)

22. Yokoyama, M., Okano, T., Sakurai, Y., Ekimoto, H., Shibazaki, C., Kataoka, K.: Toxicity and antitumor activity against solid tumors of micelle-forming polymeric anticancer drug and its extremely long circulation in blood. Cancer Res. 51, 3229-3236 (1991)

23. Couvreur, P., Kante, B., Grislain, L., Roland, M., Speiser, P.: Toxicity of polyalkylcyanoacrylate nanoparticles II: doxorubicinloaded nanoparticles. J. Pharm. Sci. 71, 790-792 (1982)

24. Thrall, J.H.: Nanotechnology and medicine. Radiology 230, 315-318 (2004)

25. Moghimi, S.M., Hunter, A.C., Murray, J.C.: Long-circulating and target-specific nanoparticles: theory to practice. Pharmacol. Rev. 53, 283 (2001)

26. Vauthier, C., Labarre, D., Ponchel, G.: Design aspects of poly(alkylcyanoacrylate) nanoparticles for drug delivery. J. Drug Target. 15, 641 (2007) 
27. Nazarov, G.V., Galan, S.E., Nazarova, E.V., Karkishchenko, N.N., Muradov, M.M., Stepanov, V.A.: Nanosized forms of drugs (a review). Pharm. Chem. J. 43, 163-170 (2009)

28. Gulyaev, A.E., Gelperina, S.E., Skidan, I.N., Antropov, A.S., Kivman, G.Y., Kreuter, J.: Significant transport of doxorubicin into the brain with polysorbate 80 -coated nanoparticles. Pharm. Res. 16, 1564-1569 (1999)

29. Han, G., Ghosh, P., Rotello, V.M.: Functionalized gold nanoparticles for drug delivery. Nanomedicine 2, 113-123 (2007)

30. Vinagradov, S.V., Bronich, T.K., Kabanov, A.V.: Pluronic block copolymers: novel functional molecules for gene therapy. Adv. Drug Del. Rev. 54, 223-233 (2002)

31. Arayne, M.S., Sultana, N., Sabah, N.: Fabrication of solid nanoparticles for drug delivery. Pak. J. Pharm. Sci. 20, 251-259 (2007)

32. Gelperina, S., Kisich, K., Iseman, M.D., Heifets, L.: The potential advantages of nanoparticle drug delivery systems in chemotherapy of tuberculosis. Am. J. Respir. Crit. Care Med. 172, 1487-1490 (2005)

33. Yamaguchi, Y., Igarashi, R.: Nanotechnology for therapy of type 2 diabetes. Nihon Rinsho 64, 295-300 (2006)

34. Akamatsu, K., Takei, S., Mizuhata, M., Kajinami, A., Deki, S., Takeoka, S., Fujii, M., Hayashi, S., Yamamoto, K.: Preparation and characterization of polymer thin films containing silver and silver sulfide nanoparticles. Thin Solid Films 359, 55-60 (2000)

35. Zeng, R., Rong, M.Z., Zhang, M.Q., Liang, H.C., Zeng, H.M.: Laser ablation of polymer-based silver nanocomposites. Appl. Surf. Sci. 187, 239-247 (2002)

36. Hussain, I., Brust, M., Papworth, A.J., Cooper, A.I.: Preparation of acrylate-stabilized gold and silver hydrosols and gold-polymer composite films. Langmuir 19, 4831-4835 (2003)

37. Kumar, R.V., Elgamiel, R., Diamant, Y., Gedanken, A., Norwig, J.: Sonochemical preparation and characterization of nanocrystalline copper oxide embedded in poly(vinyl alcohol) and its effect on crystal growth of copper oxide. Langmuir 17, 1406-1410 (2001)

38. Sajinovic, D., Saponjic, Z.V., Cvjeticanin, N., Marinovic, C.M., Nedeljkovic, J.M.: Synthesis and characterization of CdS quantum dots-polystyrene composite. Chem. Phys. Lett. 329, 168-172 (2000)

39. Kumar, R.V., Koltypin, Y., Cohen, Y.S., Aurbach, D., Palchik, O., Felner, I., Gedanken, A.: Preparation of amorphous magnetite nanoparticles embedded in polyvinyl alcohol using ultrasound radiation. J. Mater. Chem. 10, 1125-1129 (2000)

40. Yu, S.H., Yoshimura, M., Moreno, J.M.C., Fujiwara, T., Fujino, T., Teranishi, R.: In situ fabrication and optical properties of a novel polystyrene/semiconductor nanocomposite embedded with CdS nanowires by a soft solution processing route. Langmuir 17, 1700 (2001)

41. Yang, S.C., Ge, H.X., Hu, Y., Jiang, X.Q., Yang, C.Z.: Doxorubicin-loaded poly(butylcyanoacrylate) nanoparticles produced by emulsifier-free emulsion polymerization. J. Appl. Polym. Sci. 78, 517-526 (2000)

42. Huang, H., Yuan, Q., Yang, X.: Preparation and characterization of metal-chitosan nanocomposites. Colloids Surf. B 39, 31-37 (2004)

43. Egea, M.A., Gamisani, F., Valero, J., Garcia, M.E., Garcia, M.L.: Entrapment of cisplatin into biodegradable polyalkylcyanoacrylate nanoparticles. Farmaco 49, 211-217 (1994)

44. Santhi, K., Dhanaraj, S.A., Joseph, V., Ponnusankar, S., Suresh, B.: A study on the preparation and anti-tumor efficacy of bovine serum albumin nanospheres containing 5-fluorouracil. Drug Dev. Ind. Pharm. 28, 1171-1179 (2002)

45. Mu, L., Feng, S.S.: A novel controlled release formulation for the anticancer drug paclitaxel $\left(\right.$ Taxol $\left.^{\circledR}\right)$ : PLGA nanoparticles containing vitamin E TPGS. J. Control Release 86, 33-48 (2003)
46. Chawla, J.S., Amiji, M.M.: Biodegradable poly ( $\varepsilon$-caprolactone) nanoparticles for tumor-targeted delivery of tamoxifen. Int. J. Pharm. 249, 127-138 (2002)

47. Jain, R.A.: The manufacturing techniques of various drug loaded biodegradable poly(lactide-co-glycolide) (PLGA) devices. Biomaterials 21, 2475-2490 (2000)

48. Jie, Y., Wenfeng, L., Chang, Y., Chengguang, Z., Langping, J., Yili, Z., Xuzhong, X., Siyang, D., Xincheng, L., Ouchen, W.: Amphiphilically modified chitosan cationic nanoparticles for drug delivery. J. Nanopart. Res. 15, 2123 (2013)

49. Shengtang, H., Ying, W., Zheng, W., Jiliang, W.: Folate-conjugated chitosan-polylactide nanoparticles for enhanced intracellular uptake of anticancer drug. J. Nanopart. Res. 15, 2096 (2013)

50. Murugan, E., Geetha, R.D.P., Yogaraj, V.: Drug delivery investigations of quaternised poly(propylene imine) dendrimer using nimesulide as a model drug. Colloids Surf. B 114, 121-129 (2014)

51. Abdullah, G.Z., Abdulkarim, M.F., Mallikarjun, C., Mahdi, E.S., Basri, M., Abdul Sattar, M., Noor, A.M.: Carbopol 934, 940 and Ultrez 10 as viscosity modifiers of palm olein esters based nano-scaled emulsion containing ibuprofen, Pak. J. Pharm. Sci. 26, 75-83 (2013)

52. Behbehani, G.R., Soleimani, M., Khani, A., Barzegarand, L., Bagheri, S.: The effect of colloidal silica nanoparticles on the activity of $\alpha$-amylase. Pak. J. Chem. 2, 200-202 (2012)

53. Park, J.H., Cho, Y.W., Son, Y.J., Kim, K., Chung, H., Jeong, S.Y., Choi, K., Park, C.R., Park, R.W., Kim, I., Kwon, I.C.: Preparation and characterization of self-assembled nanoparticles based on glycol chitosan bearing adriamycin. Colloid Polym. Sci. 284, 763-770 (2006)

54. Soppimath, K.S., Aminabhavi, T.M., Kulkarni, A.R., Rudzinski, W.E.: Biodegradable polymeric nanoparticles as drug delivery devices. J. Control. Release 70, 1-20 (2001)

55. Tan, W., Wang, K.M., He, X., Zhao, X.J., Drake, T., Wang, L., Bagwe, R.P.: Bionanotechnology based on silica nanoparticles. Med. Res. Rev. 24, 621-638 (2004)

56. Stroh, M., Zimmer, J.P., Duda, D.G., Levchenko, T.S., Cohen, K.S., Brown, E.B., Scadden, D.T., Torchilin, V.P., Bawendi, M.G., Fukumura, D., Jain, R.K.: Quantum dots spectrally distinguish multiple species within the tumor milieu in vivo. Nat. Med. 11, 678-682 (2005)

57. Michalet, X., Pinaud, F.F., Bentolila, L.A., Tsay, J.M., Doose, S., Li, J.J., Sundaresan, G., Wu, A.M., Gambhir, S.S., Weiss, S.: Quantum dots for live cells, in vivo imaging, and diagnostics. Science 307, 538-544 (2005)

58. Daniel, M.C., Astruc, D.: Gold nanoparticles: assembly, supramolecular chemistry, quantum-size-related properties, and applications toward biology, catalysis, and nanotechnology. Chem. Rev. 104, 293-346 (2004)

59. Nichkova, M., Dosev, D., Gee, S.J., Hammock, B.D., Kennedy, I.M.: Microarray immunoassay for phenoxybenzoic acid using polymer encapsulated Eu: $\mathrm{Gd}_{2} \mathrm{O}_{3}$ nanoparticles as fluorescent labels. Anal. Chem. 77, 6864-6873 (2005)

60. Chen, Y., Chi, Y., Wen, H., Lu, Z.: Sensitized luminescent terbium nanoparticles: preparation and time-resolved fluorescence assay for DNA. Anal. Chem. 79, 960-965 (2007)

61. Parveen, S., Misra, R., Sahoo, S.K.: Nanoparticles: a boon to drug delivery, therapeutics, diagnostics and imaging, nanomedicine: nanotechnology. Biology and Medicine 8, 147-166 (2012)

62. Lee, J.E., Lee, N., Kim, H., Kim, J., Choi, S.H., Kim, J.H., Kim, T., Song, I.C., Park, S.P., Moon, W.K., Hyeon, T.: Uniform mesoporous dye-doped silica nanoparticles decorated with multiple magnetite nanocrystals for simultaneous enhanced magnetic resonance imaging, fluorescence imaging, and drug delivery. J. Am. Chem. Soc. 132, 552-557 (2010)

63. Yang, X., Li, Z., Li, M., Ren, J., Qu, X.: Fluorescent protein capped mesoporous nanoparticles for intracellular drug delivery and imaging. Chem. Eur. J. 19, 15378-15383 (2013) 
64. Li, L., Fan, M., Brown, R., Van, L.J., Wang, J., Wang, W., Song, Y., Zhang, P.: Synthesis, properties, and environmental applications of nanoscale iron-based materials: a review. Crit. Rev. Environ. Sci. Technol. 36, 405-431 (2006)

65. Kim, C.K., Kalluru, R.R., Singh, J.P., Fortner, A., Griffin, J., Darbha, G.K., Ray, P.C.: Gold-nanoparticle-based miniaturized laser-induced fluorescence probe for specific DNA hybridization detection: studies on size-dependent optical properties. Nanotechnology 17, 3085 (2006)

66. Dhar, S., Reddy, E.M., Shiras, A., Pokharkar, V., Prasad, B.L.V.: Natural gum reduced/stabilized gold nanoparticles for drug delivery formulations. Chem. Eur. J. 14, 10244-10250 (2008)

67. Selvakannan, P.R., Mandal, S., Phadtare, S., Gole, A., Pasricha, R., Adyanthaya, S.D., Murali, S.: Water-dispersible tryptophanprotected gold nanoparticles prepared by the spontaneous reduction of aqueous chloroaurate ions by the amino acid. J. Colloid Interface Sci. 269, 97-102 (2004)

68. Niemeyer, C.M.: Functional hybrid devices of proteins and inorganic nanoparticles. Angew. Chem. 42, 5796-5800 (2003)

69. Woehrle, G.H., Brown, L.O., Hutchison, J.E.: Thiol-functionalized, 1.5-nm gold nanoparticles through ligand exchange reactions: scope and mechanism of ligand exchange. J. Am. Chem. Soc. 127, 2172-2183 (2005)

70. Liu, Y., Shipton, M.K., Ryan, J., Kaufman, E.D., Franzen, S., Feldheim, D.L.: Synthesis, stability, and cellular internalization of gold nanoparticles containing mixed peptide-poly(ethylene glycol) monolayers. Anal. Chem. 79, 2221-2229 (2007)

71. Hurst, S.J., Lytton-Jean, A.K.R., Mirkin, C.A.: Maximizing DNA loading on a range of gold nanoparticle sizes. Anal. Chem. 78, 8313-8318 (2006)
72. Hwu, J.R., Lin, Y.S., Josephrajan, T., Hsu, M.H., Cheng, F.Y., Yeh, C.S., Su, W.C., Shieh, D.B.: Targeted paclitaxel by conjugation to iron oxide and gold nanoparticles. J. Am. Chem. Soc. 131, 66-68 (2009)

73. Joshi, H.M., Bhumkar, D.R., Joshi, K., Pokharkar, V., Sastry, M.: Gold nanoparticles as carriers for efficient transmucosal insulin delivery. Langmuir 22, 300-305 (2006)

74. Gu, H.W., Ho, P.L., Tong, E., Wang, L., Xu, B.: Presenting vancomycin on nanoparticles to enhance antimicrobial activities. Nano Lett. 3, 1261-1263 (2003)

75. Tom, R.T., Suryanarayanan, V., Reddy, P.G., Baskaran, S., Pradeep, T.: Ciprofloxacin-protected gold nanoparticles. Langmuir 20, 1909-1914 (2004)

76. Gibson, J.D., Bshnu, P.K., Eugene, R.Z.: Paclitaxel-functionalized gold nanoparticles. J. Am. Chem. Soc. 129, 11653-11661 (2007)

77. Mirza A.Z and Shamshad H., Preparation and characterization of doxorubicin functionalized gold nanoparticles, European Journal of Medicinal Chemistry, 46, 1857-1860 (2011)

78. Mirza A.Z., Shamshad H.: A versatile approach for the functionalization of gold nanorods and nanoparticles. J. Nanopart. Res. 15, 1-6 (2013) (art no. 1404)

79. Verhaegh, N.A.M., van Blaaderen, A.: Dispersions of rhodaminelabeled silica spheres: synthesis, characterization, and fluorescence confocal scanning laser microscopy. Langmuir 10, 1427-1438 (1994)

80. Nyffenegger, R., Quellet, C., Ricka, J.: Synthesis of fluorescent, monodisperse, colloidal silica particles. J. Colloid Interface Sci. 159, 150-157 (1993) 